University of Nebraska - Lincoln

DigitalCommons@University of Nebraska - Lincoln

$11-1-2007$

\title{
THE CASE FOR OPTICALLY THICK HIGH-VELOCITY BROAD-LINE REGION GAS IN ACTIVE GALACTIC NUCLEI
}

\author{
Stephanie A. Snedden \\ University of Nebraska-Lincoln, snedden@apo.nmsu.edu \\ C. Martin Gaskell \\ University of Nebraska-Lincoln, mgaskell@ucsc.edu
}

Follow this and additional works at: https://digitalcommons.unl.edu/physicsgaskell

Part of the Physics Commons

Snedden, Stephanie A. and Gaskell, C. Martin, "THE CASE FOR OPTICALLY THICK HIGH-VELOCITY BROAD-LINE REGION GAS IN ACTIVE GALACTIC NUCLEI" (2007). Martin Gaskell Publications. 19. https://digitalcommons.unl.edu/physicsgaskell/19

This Article is brought to you for free and open access by the Research Papers in Physics and Astronomy at DigitalCommons@University of Nebraska - Lincoln. It has been accepted for inclusion in Martin Gaskell Publications by an authorized administrator of DigitalCommons@University of Nebraska - Lincoln. 
The Astrophysical Journal, 669:126-134, 2007 November 1

(C) 2007. The American Astronomical Society. All rights reserved. Printed in U.S.A.

\title{
THE CASE FOR OPTICALLY THICK HIGH-VELOCITY BROAD-LINE REGION GAS IN ACTIVE GALACTIC NUCLEI
}

\author{
Stephanie A. Snedden ${ }^{1}$ and C. Martin Gaskell ${ }^{2}$ \\ Department of Physics and Astronomy, University of Nebraska, Lincoln, NE 68588-0111; \\ snedden@apo.nmsu.edu, mgaskell1@unl.edu \\ Received 2001 August 24; accepted 2007 June 29
}

\begin{abstract}
A combined analysis of the profiles of the main broad quasar emission lines in both Hubble Space Telescope and optical spectra shows that while the profiles of the strong UV lines are quite similar, there is frequently a strong increase in the $\mathrm{Ly} \alpha / \mathrm{H} \alpha$ ratio in the high-velocity gas. We show that the suggestion that the high-velocity gas is optically thin presents many problems. We show that the relative strengths of the high-velocity wings arise naturally in an optically thick BLR component. An optically thick model successfully explains the equivalent widths of the lines, the $\mathrm{Ly} \alpha / \mathrm{H} \alpha$ ratios and flatter Balmer decrements in the line wings, the strengths of $\mathrm{C}$ III] and the $\lambda 1400$ blend, and the strong variability in flux of high-velocity, high-ionization lines (especially $\mathrm{He}$ II and $\mathrm{He} \mathrm{I}$ ).
\end{abstract}

Subject headings: galaxies: active — galaxies: Seyfert — line: formation — line: profiles — quasars: emission lines — quasars: general

\section{INTRODUCTION}

The nature and origin of the broad-line region (BLR) in active galactic nuclei (which we will refer to simply as "quasars") has been a long-standing problem. It has been known for some time that the shapes of broad emission lines differ from line to line within the same object depending on the ionization level of the species producing each line. For example, He I $\lambda 5876$ is broader than $\mathrm{H} \alpha$ (Osterbrock \& Shuder 1982), O I $\lambda 1304$ and C II] $\lambda 2326$ are narrower than C Iv (Wilkes 1984), C iv $\lambda 1549$ tends to be broader than $\mathrm{Mg}$ II $\lambda 2798$ (Mathews \& Wampler 1985), and $\mathrm{H} \alpha$ is narrower than $\mathrm{H} \beta$ (Osterbrock 1977). $\mathrm{H} \alpha$ and $\mathrm{H} \beta$ have weaker wings than Ly $\alpha$ (Zheng 1992; Netzer et al. 1995). ${ }^{3}$ These differences in line profiles imply differences in physical conditions as a function of velocity and probably as a function of distance from the central ionizing source. One of many unresolved BLR questions is whether these differences can be explained by one BLR with a range of conditions as a function of radius, or whether there are two (or more) fundamentally distinct components. It is common to discuss two separate components, but there are differences in the literature as to how to divide the BLR into two components. In disk-plus-wind models (e.g., Chiang \& Murray 1996; Bottorff et al. 1997) the two components are identified with emission from near the disk and emission from a wind above the disk. Some observers and phenomenologists have made distinctions primarily on the basis of the mean degree of ionization, others primarily on the basis of Doppler widths. There is substantial overlap in these classifications. Gaskell (1987) and CollinSouffrin \& Lasota (1988) make a division, motivated by profile differences and photoionization modeling issues, into two components, one with a typical nebular spectrum (called "BLR I" by Gaskell 1987 and "HIL" by Collin-Souffrin \& Lasota) and one with substantial emission from clouds with large partially ionized

\footnotetext{
${ }^{1}$ Current address: Apache Point Observatory, 2001 Apache Point Road, Sunspot, NM 88349-0059.

2 Current address: Astronomy Department, University of Texas, Austin, TX 78712-0259.

3 It can be misleading to use FWHM as a measure of how broad emission lines are in the wings. Large contributions to the core can skew the FWHM to lower values. For example, although the line profile ratios reveal that the Ly $\alpha$ wings are broader, Table 1 of Netzer et al. (1995) shows that $\mathrm{FWHM}_{\mathrm{H} \beta}>\mathrm{FWHM}_{\mathrm{Ly} \alpha}$.
}

zones ("BLR II" or "LIL"). The case for two such components is summarized by Gaskell (2000). Other workers, motivated by analyses of line profiles, have separated the BLR into a "very broad line region" (VBLR) and another component. The other component has been called the "intermediate-line region" (ILR) by Wills et al. (1993) and Brotherton et al. (1994) or the "classical broad component" (BC) by Sulentic et al. (2000). Note that the $\mathrm{BC}$ and ILR are not identical, and hence the corresponding VBLR components can be quite different. The VBLR, ILR, and $\mathrm{BC}$ show both high- and low-ionization emission. Despite these differing terminologies, there is general agreement that the conditions of the highest velocity gas (which we call the VBLR) are different from the other components, although the VBLR gas might merely be an extension of the other gas. Historically, photoionization models assumed that the line-emitting gas was optically thick. However, several authors have argued for a significant optically thin component to the VBLR gas, and to date there has been no resolution to this debate. The main arguments for an optically thin VBLR have been as follows:

1. The general symmetry of Lya.-Wilkes \& Carswell (1982) pointed out that BLR clouds could not be both optically thick and have a net radial motion (e.g., outflow) because Ly $\alpha$ profiles are symmetric and similar to C IV profiles. Since the blueshifting of the high-ionization lines requires at least some radial motions (Gaskell 1982), the relative symmetry of Ly $\alpha$ could mean that the VBLR (highest velocity gas associated with the profile wings) has to be optically thin.

2. An apparent lack of variability of the VBLR in Mrk 590 between two epochs.-Ferland et al. (1990) found that the VBLR Balmer emission line flux in Mrk 590 changed little between two epochs about $3 \mathrm{yr}$ apart, even though the continuum and line cores changed. Hydrogen recombination lines should change little if the VBLR is optically thin and hence fully ionized. See Marziani et al. (2003) and Korista \& Goad (2004) for additional discussion.

3. Higher $\mathrm{Ly} \alpha / \mathrm{H} \beta$ ratio for the VBLR. - Zheng (1992) showed that for some AGNs the $\mathrm{Ly} \alpha / \mathrm{H} \beta$ ratio is much higher in the wings and that it approached the Menzel-Baker case B value that might be expected from optically thin gas.

4. Emission-line responses might require negative responsivities.-Sparke (1993) pointed out that the shapes of some of 

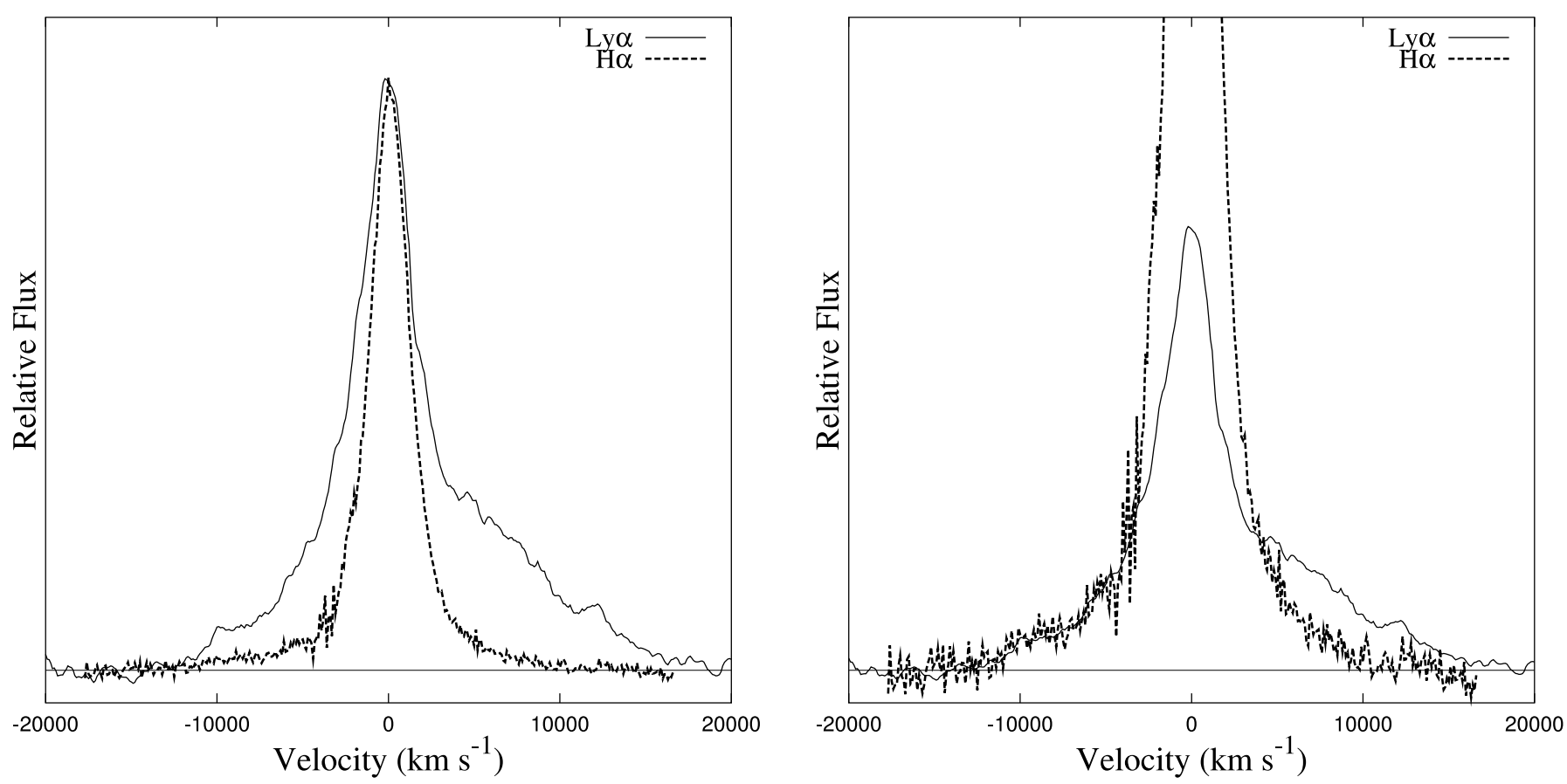

FIG. 1. - Ly $\alpha$ profile in PG $1116+215$, with no deblending, compared with the H $\alpha$ profile in the same object. [N II] has been removed from the H $\alpha$ profile. Left: The two lines normalized to the same peak; right: the lines normalized so that the short-wavelength wings match. The profile differences at +5000 to $+10,000 \mathrm{~km}{ }^{-1}$ mostly likely have a significant contribution from $\mathrm{N}$ v and $\mathrm{Si}$ II emission.

the line-continuum cross-correlation functions from 1989 monitoring of NGC 5548 seem to require the emission of the inner BLR to decline as the continuum increases. She suggested that optically thin clouds in the inner BLR could do this.

The advent of the Hubble Space Telescope (HST) makes possible the comparison of high-quality line profiles of both the highionization UV lines and the Balmer lines in the same objects. In this paper we use such comparisons to investigate the nature of the VBLR, and we argue that the bulk of the VBLR is in fact not optically thin.

\section{DATA ANALYSIS}

We have determined the $\mathrm{Ly} \alpha / \mathrm{H} \alpha$ ratio as a function of velocity for eight AGNs with high signal-to-noise ratio (S/N) HST FOS and optical spectra. Stirpe $(1990,1991)$ presented high-quality Balmer line profiles for bright low-redshift AGNs. For this study we have chosen eight of these that also had high S/N HST FOS spectra available. Six out of these eight AGNs are radio-quiet.

\subsection{Flux Levels}

The objects in the sample were not observed simultaneously in the UV and optical bandpasses, although this only introduces an uncertainty in the ratios at the $20 \%$ level and does not affect our conclusions. As a check of the effect of variability in emissionline flux on our sample, the line ratios of seven of our objects were compared with integrated line fluxes from the literature that were measured at different times. Six of these were found to be the same to within $20 \%$. Another, PKS $2251+113$, showed a $20 \%$ change in flux compared with Espey et al. (1994) and a 30\% change compared with Boroson \& Green (1992). The remaining object, B2 2201+315 (=4C 31.36), had been subject to long-term broadband optical monitoring, and optical continuum variations were less than $5 \%$. Line variations will be less than this. We also compared $U V$ lines of NGC 5548 at high and low states during the International AGN Watch campaign, with lines in the optical spectra taken at one (different) epoch, and found only a $10 \%$ difference in the predicted physical conditions of the BLR gas. Since the optical spectra were obtained with a narrow slit, we determined and applied a small $(17 \%)$ correction to the optical line fluxes. This value was obtained by comparing the Stirpe sample's integrated Balmer line fluxes with those in the literature and has a negligible effect on our results here.

\subsection{Line Profiles}

Determining the profiles of the broad emission lines in AGNs is difficult. Two issues that particularly affect the line profile are (1) the subtraction of the appropriate continuum level and (2) the deblending of weaker lines.

\subsubsection{The Effects of Continuum Level Placement}

Errors in line profiles due to continuum-level placement only have a significant effect in the high-velocity wings of the lines. In Figures $1 a$ and $1 b$ we show a comparison of $\operatorname{Ly} \alpha$ and $\mathrm{H} \alpha$ for a typical object. In this paper we only derive physical conditions at five projected velocities: $0, \pm 5000$, and $\pm 10,000 \mathrm{~km} \mathrm{~s}^{-1}$. Assuming that the short wavelength wing of $\operatorname{Ly} \alpha$ is uncontaminated, it is possible to see by comparing the wings at $\pm 10,000 \mathrm{~km} \mathrm{~s}^{-1}$ in Figure $1 b$ that the combined errors of continuum placement and incomplete deblending on the red side of Ly $\alpha$ are $\sim 20 \%$. This is typical for most of our objects, is comparable to the uncertainty due to counting statistics, and is unimportant for our analyses. The wings of the Balmer lines are considerably weaker in all our objects, and as can be seen from Figure 1, the continuum placement is clearly more critical. The most problematic line is $\mathrm{H} \beta$, and Shuder (1982) has illustrated in his Figure 2 the effects of different choices of local continuum. The line for which it is easiest to define an underlying continuum is $\mathrm{H} \alpha$, but as can be seen in our Figure $1 a$, the $\mathrm{H} \alpha$ flux at $\pm 10,000 \mathrm{~km} \mathrm{~s}^{-1}$ can be uncertain by a factor of 2 . In general, $\mathrm{H} \alpha$ is very symmetric in our objects 
and the consistency we get in flux ratios between the two wings for our sample as a whole suggests that possible errors in the choice of the continuum are not a major issue.

\subsubsection{Deblending}

The degree of blending varies from line to line. The least problematic line is $\mathrm{H} \alpha$. It is only blended with the narrow [ $\mathrm{N}$ II] lines, which are easy to deal with. Apart from occasional narrow absorption lines, $\operatorname{Ly} \alpha$ is free of blending with other emission lines on the short wavelength side, but on the long wavelength side it is blended with $\mathrm{N} v \lambda 1240$ and $\mathrm{Si}$ II $\lambda 1265$. These blends cover our +5000 to $+10,000 \mathrm{~km} \mathrm{~s}^{-1}$ velocity range. The line with most serious blending problems is $\mathrm{H} \beta$. The narrow but strong [O III] lines occur at +6000 and $+9000 \mathrm{~km} \mathrm{~s}^{-1}$ in the red wing of $\mathrm{H} \beta$, and the most problematic blend is the broad He II $\lambda 4686$, which occurs at $-11,000 \mathrm{~km} \mathrm{~s}^{-1}$ and therefore contributes significantly to our $-10,000 \mathrm{~km} \mathrm{~s}^{-1}$ region. Many weak Fe II lines can also be a factor. The effects of uncertainties in continuum placement and deblending for $\mathrm{H} \beta$ have been extensively investigated by Shuder (1982, 1984) and Crenshaw (1986).

The deblending of the Balmer lines in the objects we consider was performed by G. Stirpe and is discussed in detail in Stirpe (1990), where raw and deblended profiles can be seen for all objects. Briefly, after the spectra of the Balmer lines were fluxcalibrated and corrected for atmospheric absorption features, they were fit with a power-law continuum. Blended emission lines were removed from the Balmer lines, and the narrow-line components of $\mathrm{H} \alpha$ and $\mathrm{H} \beta$ were removed, all as detailed in that paper. We deblended $\mathrm{N} v \lambda 1240$ and Si II $\lambda 1265$ from the Ly $\alpha$ region with multiple Gaussians using the SPECFIT and SPLOT tasks in IRAF. The rest wavelengths were fixed but the line intensities and widths were unconstrained. We used one or two Gaussians per line. This method necessarily assumes that the lines are symmetric. A real excess in the red wing of Ly $\alpha$ is hard to distinguish from $\mathrm{N} v$ and $\mathrm{Si}$ II emission. This is a fundamental limitation of all deblending. In Figures $1 a$ and $1 b$ we show the Ly $\alpha$ region with no deblending at all. This represents a worst-case scenario for deblending. It can be seen that while the overall profiles of $\mathrm{H} \alpha$ and Ly $\alpha$ are fundamentally different, there is good agreement in the short wavelength wings, where deblending is not an issue. On the red side, where blending with $\mathrm{N} v$ and $\mathrm{Si}$ II is a problem, the profiles disagree somewhat. Although the true Ly $\alpha$ profile on the red side is unknowable, it cannot be higher than the observed profile. Since we know that there is $\mathrm{N} v$ and $\mathrm{Si}$ II in AGNs (e.g., Vanden Berk et al. 2001), the true Ly $\alpha$ profile in the red wings must be lower than the observed. As can be seen from Figure 1 , the maximum error in flux at $10,000 \mathrm{~km} \mathrm{~s}^{-1}$ in the red wing is therefore about a factor of 2 . After removing Gaussian-fit estimates of the contribution from N v $\lambda 1240$ and Si II $\lambda 1265$, the error should be less than a factor of 2 . Furthermore, the consistency of the physical conditions we deduce for the red and blue wings suggests that the error in deblending the red wing of Ly $\alpha$ is much less than a factor of 2 .

\subsection{Reddening}

Since we consider $\mathrm{Ly} \alpha / \mathrm{H} \alpha$ ratios, reddening effects are significant and corrections are required. For Galactic reddenings we adopted the average of the reddenings given by Burstein \& Heiles (1982) and Schlegel et al. (1998). We give these in Table 1. In addition, there is probably internal reddening in the AGNs themselves. De Zotti \& Gaskell (1985) found typical reddenings of $E(B-V) \sim 0.25$ for Seyfert 1 galaxies. Gaskell et al. (2004) and Gaskell \& Benker (2007) have shown that the nuclear reddening curve for AGNs is flatter than the standard Galactic red-
TABLE 1

Galactic Reddening

\begin{tabular}{|c|c|}
\hline Object & $E(B-V)$ \\
\hline 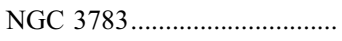 & 0.120 \\
\hline NGC 5548.................................. & 0.011 \\
\hline PG $1351+64 \ldots \ldots \ldots \ldots$. & 0.014 \\
\hline 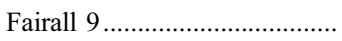 & 0.014 \\
\hline PG $1211+143 \ldots \ldots \ldots \ldots \ldots \ldots$ & 0.034 \\
\hline PG $1116+215 \ldots \ldots \ldots \ldots \ldots$ & 0.012 \\
\hline B2 2201+31 .............................. & 0.015 \\
\hline PKS $2251+11 \ldots \ldots \ldots \ldots \ldots \ldots$ & 0.064 \\
\hline
\end{tabular}

dening curve in the UV. They find typical reddenings of $E(B-V)$ from $0.1-0.4$ in the optical. These ranges agree with $E(B-V) \sim$ 0.25 , as found by Ward \& Morris (1984) for several line ratios of NGC 3783 (i.e., 0.13 greater than the Galactic reddening in Table 1). Netzer et al. (1995) and Bechtold et al. (1997) found that the $\mathrm{Ly} \alpha / \mathrm{H} \alpha$ ratio for quasars was correlated with the $0.1-0.6 \mu \mathrm{m}$ flux ratio. For a typical quasar this implies a reddening of $E(B-V) \sim 0.15$ (including the Galactic contribution). By comparing continuum shapes from $0.1-0.9 \mu \mathrm{m}$, Gaskell \& Benker (2007) find a median internal $E(B-V)$ of 0.08 mag for the 17 UV-bright quasars they consider. We therefore believe that the total reddenings of our sample could have a Galactic reddening equivalent of 0.1 or more greater than those given in Table 1 (assuming a standard Galactic reddening curve). In Table 2 we show Galactic reddening-corrected $\mathrm{Ly} \alpha / \mathrm{H} \alpha$ ratios at $\pm 10,000$, $\pm 5000, \pm 2500$, and $0 \mathrm{~km} \mathrm{~s}^{-1}$ for our sample. ${ }^{4}$ The ratios are averaged over $\pm 1000 \mathrm{~km} \mathrm{~s}^{-1}$. In Table 3 we show the $\mathrm{H} \beta / \mathrm{H} \alpha$ ratios at the same velocities. The emission lines were deblended as described in $\S 2.2 .2$ before the reddening corrections were applied. The most uncertain values are those at $+10,000 \mathrm{~km} \mathrm{~s}^{-1}$, where the incomplete deblending of $\mathrm{N} v$ causes problems for Ly $\alpha$ and $[\mathrm{O}$ III] causes problems for $\mathrm{H} \beta$. Plots of the $\mathrm{Ly} \alpha / \mathrm{H} \alpha$ ratio profiles for three individual objects can be seen in Snedden \& Gaskell (1997, 1999b), but these plots were not reddening-corrected and the vertical scale was calculated incorrectly (it is too large by a factor of $6563 / 1216=5.4$ ). In Figures $2 a$ and $2 b$ we show the average $\mathrm{Ly} \alpha / \mathrm{H} \alpha$ and $\mathrm{H} \alpha / \mathrm{H} \beta$ ratios for our entire sample as a function of velocity. These values are taken from Tables 2 and 3. To illustrate the uncertainty due to the reddening correction, we show (as smooth curves) the effect of dereddening by an additional $E(B-V)=0.1$. It is possible in some objects that the actual dereddening needed could be even greater, although Gaskell et al. (2004) find that the average reddening of high-luminosity AGNs is low.

\section{PHOTOIONIZATION MODELS}

It has long been known that the hydrogen density, $n_{\mathrm{H}}$, and the ionization parameter, $U, 5$ of quasar BLRs can be determined from the relative strengths of the C IV $\lambda 1549$, Ly $\alpha$, and C III] $\lambda 1909$ lines (e.g., Davidson 1977; Mushotzky \& Ferland 1984). We have generated grids of line intensity ratios for these emission lines from models calculated using the photoionization code CLOUDY, version C90.04 (Ferland 2002). In CLOUDY ionization equilibrium and thermal equilibrium are solved for in zones

\footnotetext{
${ }^{4}$ Note that NGC 3783, for which Ward \& Morris (1984) get a higher reddening, has the lowest $\mathrm{Ly} \alpha / \mathrm{H} \alpha$ ratio.

5 The ionization parameter is defined as $U=\Phi(\mathrm{H}) /[n(\mathrm{H}) \times c]$, where $\Phi(\mathrm{H})$ is the surface flux of ionizing photons $\left(\mathrm{cm}^{-2} \mathrm{~s}^{-1}\right), n(\mathrm{H})$ is the total hydrogen density (ionized, neutral, and molecular), and $c$ is the speed of light.
} 
TABLE 2

Ly $\alpha / \mathrm{H} \alpha$ Line Ratios at Selected Velocities Corrected for Galactic Reddening

\begin{tabular}{|c|c|c|c|c|c|c|c|}
\hline Object & $\begin{array}{c}-10,000 \\
\left(\mathrm{~km} \mathrm{~s}^{-1}\right)\end{array}$ & $\begin{array}{c}-5000 \\
\left(\mathrm{~km} \mathrm{~s}^{-1}\right)\end{array}$ & $\begin{array}{c}-2500 \\
\left(\mathrm{~km} \mathrm{~s}^{-1}\right)\end{array}$ & $\begin{array}{c}0 \\
\left(\mathrm{~km} \mathrm{~s}^{-1}\right)\end{array}$ & $\begin{array}{c}2500 \\
\left(\mathrm{~km} \mathrm{~s}^{-1}\right)\end{array}$ & $\begin{array}{c}5000 \\
\left(\mathrm{~km} \mathrm{~s}^{-1}\right)\end{array}$ & $\begin{array}{c}10,000 \\
\left(\mathrm{~km} \mathrm{~s}^{-1}\right)\end{array}$ \\
\hline 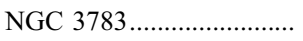 & 4.6 & 4.4 & 1.4 & 1.3 & 2.4 & 5.3 & .. \\
\hline 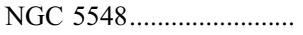 & $\ldots$ & 1.8 & 0.44 & 1.5 & 0.89 & 2.1 & $\ldots$ \\
\hline PG $1351+64 \ldots \ldots \ldots \ldots \ldots \ldots$ & 11.3 & 10.4 & 9.6 & 6.0 & 5.2 & 3.8 & 7.1 \\
\hline Fairall $9 \ldots \ldots \ldots \ldots$ & 4.5 & 4.4 & 4.2 & 6.0 & 3.0 & 8.3 & 11.9 \\
\hline 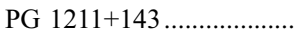 & 2.0 & 4.8 & 3.1 & 2.6 & 2.6 & 5.6 & 5.4 \\
\hline PG $1116+215 \ldots \ldots \ldots \ldots$ & 16.1 & 16.4 & 8.7 & 3.4 & 6.1 & 15.0 & $\ldots$ \\
\hline B2 $2201+31 \ldots \ldots \ldots \ldots \ldots$ & 7.0 & 5.6 & 7.3 & 3.0 & 4.0 & 4.7 & 8.2 \\
\hline PKS $2251+11 \ldots \ldots \ldots \ldots \ldots \ldots$ & 4.7 & 3.2 & 2.1 & 1.8 & 1.4 & 2.3 & 5.4 \\
\hline
\end{tabular}

of approximately constant conditions. On the side of the cloud where the ionizing radiation enters, hydrogen is essentially completely ionized. When ionizing photons are no longer reaching a zone, the fraction of neutral hydrogen quickly rises (see Osterbrock 1989). The zone where this occurs corresponds to the classical Strömgren depth. The presence of X-rays in an AGN makes the transition from hydrogen being fully ionized to being largely neutral less abrupt in a BLR cloud than in a classic $\mathrm{H}$ II region or planetary nebula. We have therefore defined the Strömgren depth in a BLR cloud to be where the ionization fraction of hydrogen falls to 95\% (Snedden \& Gaskell 1999a). In CLOUDY C90 this corresponds to about 200 zones. In the models presented here we used the standard quasar continuum of Ferland (2002). For all calculations solar abundances were used. As we have shown in Snedden \& Gaskell (1999a), enhanced abundances change the ionization structure, with the result that a given $\mathrm{C}$ III]/C IV ratio corresponds to a higher density. This does not change any of the conclusions presented here. We ran grids of both optically thick and optically thin models. Other details of the models are as in Ferland \& Persson (1989).

The optically thick calculations were stopped at a column density of $10^{25.5} \mathrm{~cm}^{-2}$, as in Ferland \& Persson (1989). To see how line ratios behaved in the optically thin limit, we ran a model that stopped after only three zones (Lyman continuum optical depth, $\tau_{912} \sim 10^{-4}$ ). Although illustrative, such an extreme model has so little gas that even with a $100 \%$ covering factor it cannot produce significant emission. In order to produce the minimum observed $W_{\lambda}=50 \AA$ for the broad component of Ly $\alpha$, assuming a covering factor of $50 \%$, we find that we need $\tau_{912} \sim 1.0$. We therefore ran a third set of models that stopped at $\tau_{912}=1$. In Figures 3 and 4 we show grids of the ratios of $\mathrm{Ly} \alpha / \mathrm{C}$ IV/C III] and $\mathrm{Ly} \alpha / \mathrm{H} \alpha / \mathrm{H} \beta$ as functions of $U$ and $n_{\mathrm{H}}$ for optically thin, optically thick, and $\tau_{912}=1$ clouds. It should be noted that there can be uncertainties in line and continuum transfer that are difficult to estimate, since CLOUDY uses escape probabilities instead of more formally correct calculations (Ferland 2002). At the high densities and pressures of the BLR, this can be a serious source of error. Fortunately, the approximation is known to be exact if physical conditions are held constant over the region of interest. Since we consider emission-line ratios as a function of velocity, rather than ratios integrated across the velocity field, we are much more likely to meet this criterion. As has long been recognized (e.g., Davidson 1977), most of the strong UV lines in quasar spectra can be explained by a "canonical" density of $10^{10} \mathrm{~cm}^{-3}$ and canonical ionization parameter of the order 0.1. "Locally optimally emitting cloud" (LOC) scenarios (Baldwin et al. 1995; see also Bottorff \& Ferland 2001) can fit many line ratios by combining different clouds with different properties. By considering a single zone and the major recombination lines, however, we can compare optically thick and optically thin clouds unambiguously. Furthermore, the simplest case, that of single-zone models, matches these strong lines well.

\section{HYDROGEN LINE RATIOS}

Zheng (1992) suggests that an optically thin component is one way of getting a higher $\mathrm{Ly} \alpha / \mathrm{H} \alpha$ ratio in the wings. As discussed in $\S 3$, our very optically thin model fails to reproduce the observed $W_{\lambda}$. Furthermore, from Figure $3 a$ and the $\mathrm{Ly} \alpha / \mathrm{H} \alpha$ ratios in Table 2, it can be seen that the ratios predicted by this thinlimit model are in fact too high for both the wings and core, and could only be explained by either extremely high values of $U$ or extreme intrinsic reddening. If we use the flat nuclear reddening curve found by Gaskell et al. (2004), $E(B-V) \sim 1.0$ would be required. The Balmer decrements in the three-zone optically thin model are very uniform (close to case B) and do not match the observed values in the wings, where the decrement is quite flat (i.e., $\mathrm{H} \beta$ is too strong), unless the errors in continuum subtraction are at the $100 \%$ level, the high end of Stirpe's (1991) error

TABLE 3

H $\alpha / \mathrm{H} \beta$ Line Ratios at Selected Velocities Corrected for Galactic Reddening

\begin{tabular}{|c|c|c|c|c|c|c|c|}
\hline Object & $\begin{array}{c}-10,000 \\
\left(\mathrm{~km} \mathrm{~s}^{-1}\right)\end{array}$ & $\begin{array}{c}-5000 \\
\left(\mathrm{~km} \mathrm{~s}^{-1}\right)\end{array}$ & $\begin{array}{c}-2500 \\
\left(\mathrm{~km} \mathrm{~s}^{-1}\right)\end{array}$ & $\begin{array}{c}0 \\
\left(\mathrm{~km} \mathrm{~s}^{-1}\right)\end{array}$ & $\begin{array}{c}2500 \\
\left(\mathrm{~km} \mathrm{~s}^{-1}\right)\end{array}$ & $\begin{array}{c}5000 \\
\left(\mathrm{~km} \mathrm{~s}^{-1}\right)\end{array}$ & $\begin{array}{c}10,000 \\
\left(\mathrm{~km} \mathrm{~s}^{-1}\right)\end{array}$ \\
\hline 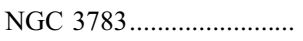 & 0.89 & 1.2 & 2.7 & 3.6 & 2.8 & 1.6 & $\ldots$ \\
\hline 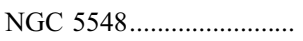 & 1.3 & 2.2 & 3.7 & 4.0 & 4.4 & 2.9 & $\ldots$ \\
\hline PG $1351+64$ & $\ldots$ & 2.0 & 2.8 & 3.3 & 2.8 & 2.6 & 2.1 \\
\hline 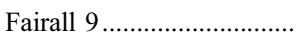 & 2.0 & 3.8 & 3.1 & 5.0 & 4.5 & 2.6 & 1.1 \\
\hline 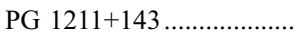 & 1.1 & 3.8 & 2.2 & 3.3 & 2.4 & 2.0 & $\ldots$ \\
\hline PG $1116+215 \ldots \ldots \ldots \ldots$ & 0.69 & 2.3 & 2.2 & 3.1 & 2.6 & 1.9 & $\ldots$ \\
\hline 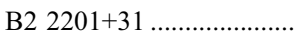 & 1.8 & 4.0 & 3.3 & 3.8 & 4.0 & 3.4 & 2.0 \\
\hline PKS $2251+11 \ldots \ldots \ldots \ldots \ldots \ldots$ & $\ldots$ & 3.8 & 4.4 & 4.5 & 4.2 & 2.4 & 1.6 \\
\hline
\end{tabular}



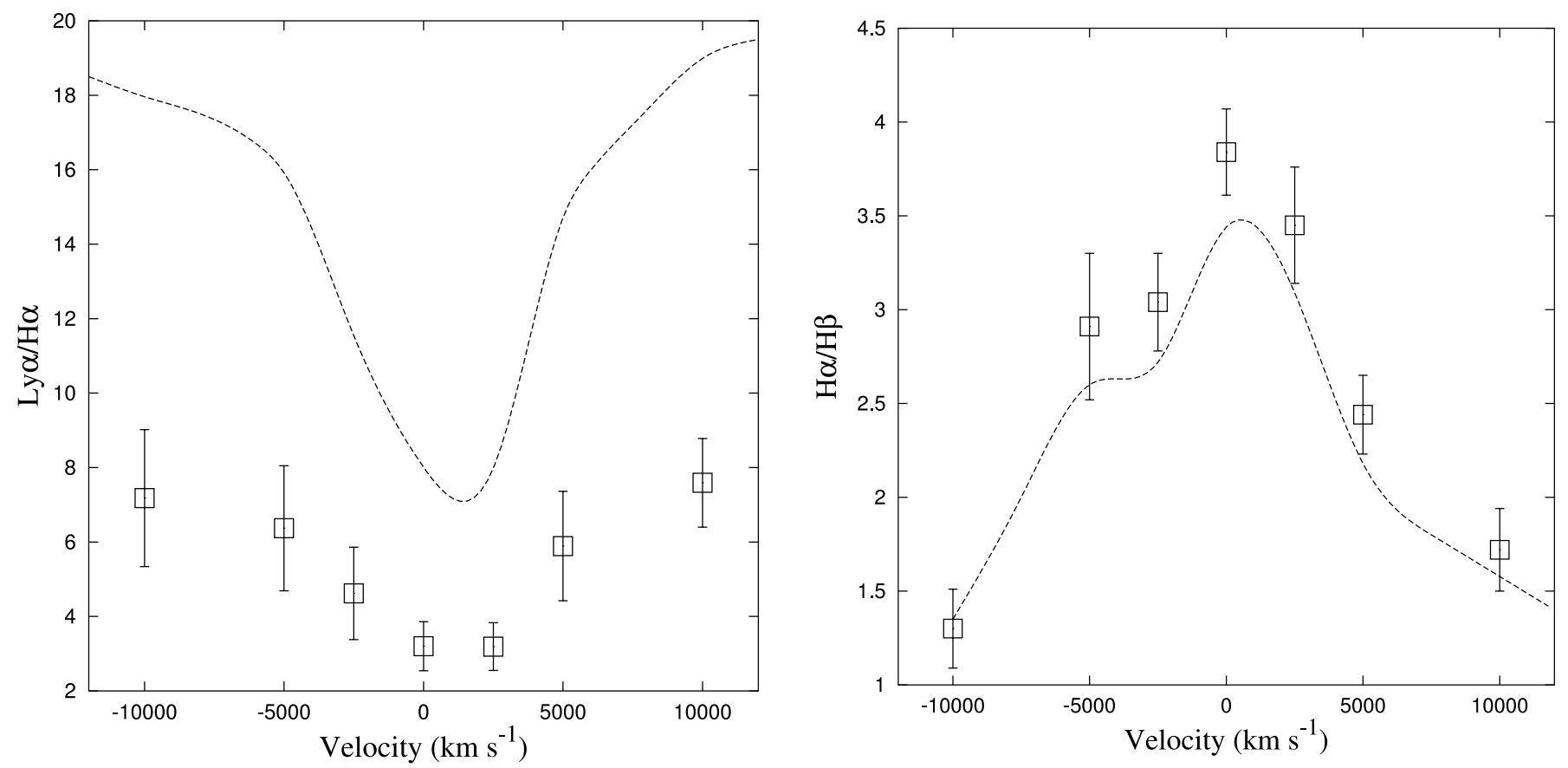

FIG. 2.-Observed hydrogen line ratios. Left: Average values of $\mathrm{Ly} \alpha / \mathrm{H} \alpha$ across the line profile for our sample are shown as boxes. These points are corrected for Galactic reddening only. The dashed curve shows $\mathrm{Ly} \alpha / \mathrm{H} \alpha$ after an additional intrinsic dereddening corresponding to $E(B-V) \approx 0.1$. Right: Same as left, but for the $\mathrm{H} \alpha / \mathrm{H} \beta$ ratio.

estimate. As has been noted by Shuder $(1982,1984)$ and Crenshaw (1986), determination of the flux in the wings of $\mathrm{H} \beta$ is very uncertain. If the continuum is indeed set too low, we can match the thin-limit model predictions in the wings but cannot match the $\mathrm{Ly} \alpha / \mathrm{H} \alpha$ ratio assuming the same physical conditions. If the error in the Balmer decrement is in fact less than a factor of 2 , we cannot match any of the observed hydrogen lines to the three-zone model.

The $\tau_{912}=1$ models fit the $\mathrm{Ly} \alpha / \mathrm{H} \alpha$ ratios in the high velocity wings and also match the lowest observed $W_{\lambda}$ if we assume a large covering factor $(50 \%)$. However, they cannot match the observed ratios for the line cores unless reddening is extreme. These models are, of course, close to being optically thick.

The optically thick model is the best match to both observed line ratios and $W_{\lambda}$ over the entire emission-line profile from -5000 to $+5000 \mathrm{~km} \mathrm{~s}^{-1}$. The oval in Figure $3 b$ shows the region in physical parameter space that matches the observed emissionline ratios. We note that it is not possible to simultaneously match $\mathrm{Ly} \alpha, \mathrm{H} \alpha$, and $\mathrm{H} \beta$ at $\pm 10,000 \mathrm{~km} \mathrm{~s}^{-1}$ with any set of models. We believe this could be a result of an oversubtraction of the continuum at $\mathrm{H} \beta$.

We cannot rule out a contribution to the wings from gas with $\tau_{912} \approx 1$, but we reiterate that this set of models requires a large covering factor and in fact approaches the optically thick case. Furthermore, we show in $\S 7$ that these same models lead us to a similar conclusion regarding the $\mathrm{C}$ III] and $\mathrm{C}$ IV emission lines. In this case, the photoionization models support an optically thick ILR and a VBLR that could have an optically thin component or be optically thick. When the overall symmetry of the ILR component of Ly $\alpha$ is considered (see Fig. 1 of Brotherton et al. 1994), it is reasonable to conclude that there is a negligible nonvirial motion of the optically thick gas. This is consistent with the results of reverberation-mapping studies (Gaskell 1988; Koratkar \& Gaskell 1991) and a necessary assumption in using such studies to calculate black hole masses.

We believe the simplest explanation is that the gas responsible for the high-velocity wings of the hydrogen emission lines con- tains a significant optically thick component, although some optically thin gas could also be present.

\section{PROFILES OF LOW-IONIZATION LINES}

$\mathrm{H} \beta$ is produced both as a recombination line in the highly ionized zone of clouds and as a collisionally excited line in the partially ionized zone. An optically thin cloud lacks the partially ionized zone and will not produce low-ionization emission lines such as Fe II, Mg II, O I, etc. If we assume that a high-ionization line includes an optically thin, high-velocity component, the profile of another line arising only from the optically thick, partially ionized zone will have a different, narrower profile. Unfortunately, there is no strong uncontaminated low-ionization line for comparison with $\mathrm{H} \beta$. The strongest low-ionization line is $\mathrm{Mg}$ II 22798. Comparisons of the wings of $\mathrm{Mg}$ II and $\mathrm{H} \beta$ are complicated by the Fe II emission around $\mathrm{Mg}$ II, but Gaskell \& Mariupolskaya (2002) find the FWHMs of $\mathrm{Mg}$ II and $\mathrm{H} \beta$ to be well-correlated for all radio classes. They find no systematic difference in the FWHMs. Detailed profile comparisons (Grandi \& Phillips 1979) are consistent with $\mathrm{Mg}$ II and $\mathrm{H} \beta$ having identical profiles. Although much weaker than $\mathrm{Mg}$ II, and in a spectral region much affected by telluric features, O I 28446 is relatively free from contamination. Morris \& Ward (1989) made profile comparisons between $\mathrm{H} \alpha$ and O I $\lambda 8446$ to search for the presence of optically thin material. Although their comparisons have been quoted as supporting the existence of optically thin material, the majority of their objects have identical $\mathrm{H} \alpha$ and $\mathrm{O}_{\mathrm{I}} \lambda 8446$ profiles, and they state that "optically thin material contributes a negligible amount of the $\mathrm{H} \alpha$ material." Their two clearest cases of profile differences are both objects showing complex line structure that we have argued elsewhere (Gaskell \& Snedden 1999) is caused by emission from a disk component. The small differences between the $\mathrm{H} \alpha$ and $\mathrm{O}_{\mathrm{I}} \lambda 8446$ profiles clearly arise from this disk component. It must have conditions producing a slightly different $\mathrm{H} \alpha / \mathrm{O}$ I $\lambda 8446$ ratio. Rodriguez-Ardila et al. (2000) find similar $\mathrm{H} \alpha$ and $\mathrm{O}_{\mathrm{I}} \lambda 8446$ profiles for all but one of the AGNs they study, and they also conclude that optically thin gas with a different velocity field (e.g., a VBLR) contributes a negligible 

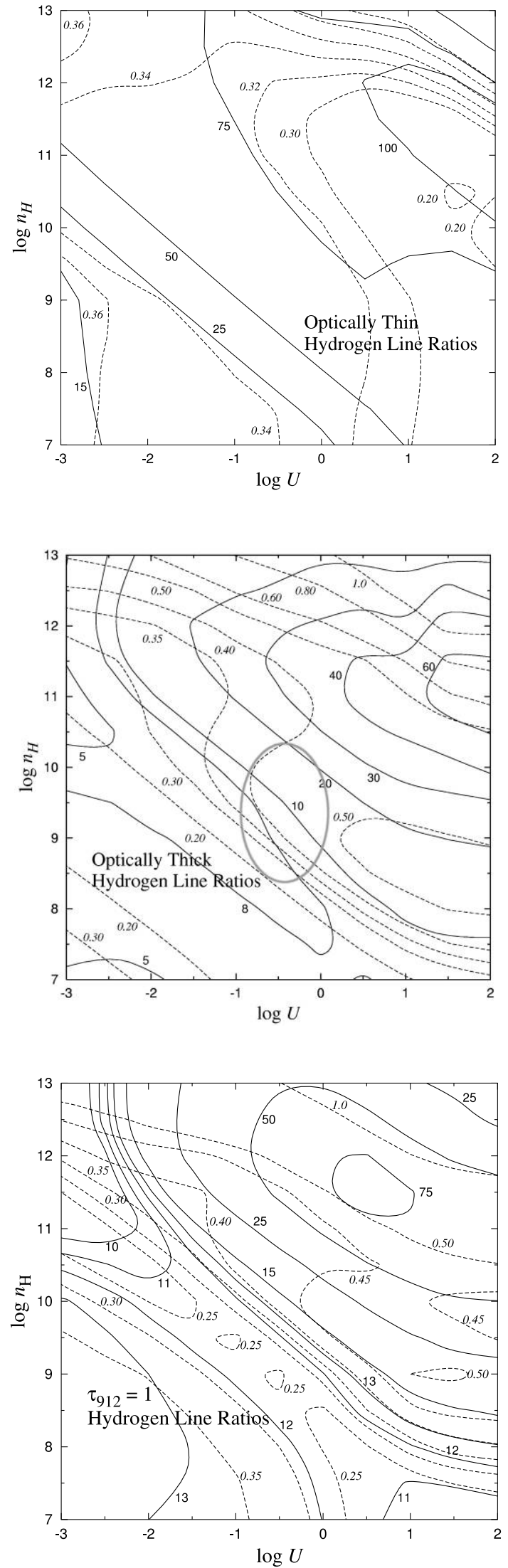

FIG. 3.- Grids of modeled hydrogen line ratios. Left: Optically thin photoionization model. Solid lines show contours of $\mathrm{Ly} \alpha / \mathrm{H} \alpha$; dashed lines show $\mathrm{H} \beta / \mathrm{H} \alpha$. We use $\mathrm{H} \beta / \mathrm{H} \alpha$ here to avoid confusion with the values for $\mathrm{Ly} \alpha / \mathrm{H} \alpha$. Center: Same as left, but for optically thick gas. The average ratios of our sample at \pm 5000 and $0 \mathrm{~km} \mathrm{~s}^{-1}$ fall inside the marked ellipse. Right: Same as left, but for a model that stops at $\tau_{912}=1$, yielding the minimum reasonable $W_{\lambda}$ for the Ly $\alpha$ broad emission-line component.
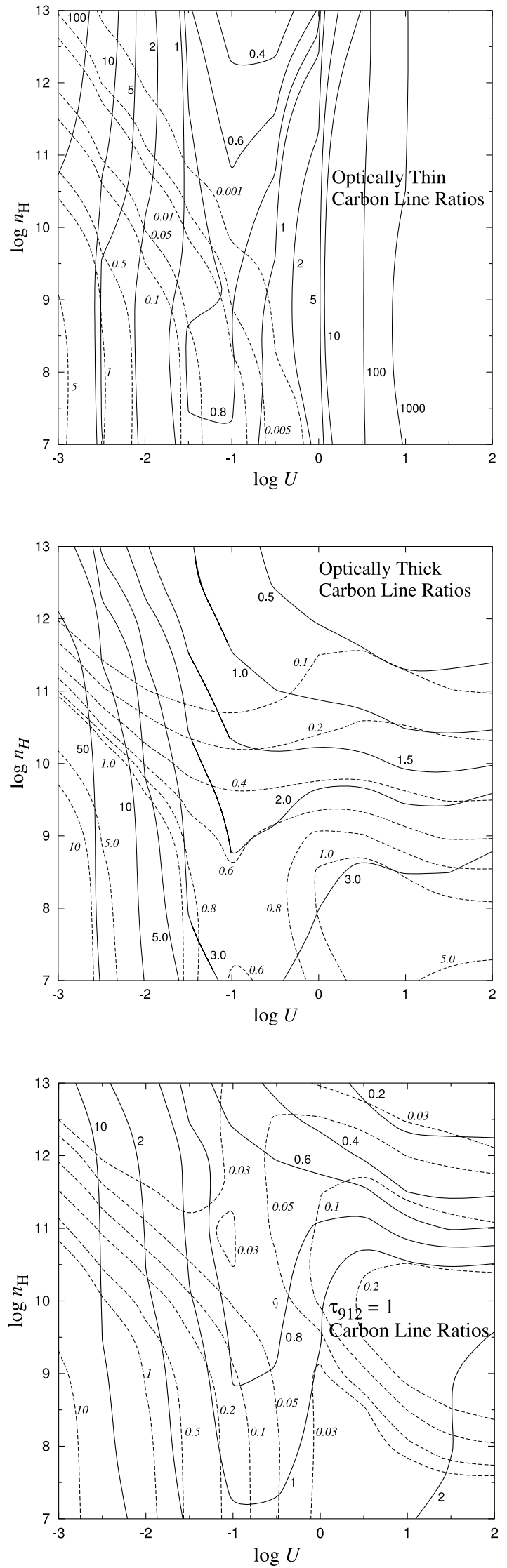

FIG. 4.-Grids of modeled carbon line ratios. Left: Optically thin photoionization model. Solid lines show contours of $\mathrm{Ly} \alpha / \mathrm{C}$ iv; dashed lines show $\mathrm{C}$ m] $/ \mathrm{C}$ Iv. Center: Same as left, but for optically thick gas. Right: Same as left, but for a model that stops at $\tau_{912}=1$, yielding the minimum reasonable $W_{\lambda}$ for the Ly $\alpha$ broad emission-line component. 
amount of $\mathrm{H} \alpha$ emission. We conclude that there is no convincing evidence from the profiles of low-ionization emission lines for a significant contribution to the line flux from optically thin gas.

\section{VARIABILITY OF HELIUM LINES}

Although lack of variability in flux of the wings of the Balmer lines between two epochs was the motive for Ferland et al. (1990) proposing that the highest velocity gas was optically thin, difference and rms spectra show that the most variable broad-line component is the high-ionization component. This can be seen, for example, in the rms spectrum of NGC 7469 (see Fig. 2 in Collier et al. 1998) and in many other rms spectra (e.g., see Figs. 1-3 in Peterson et al. 1998). The variable component of He II $\lambda 4686$ is always very broad, making it a quintessential VBLR line. In the $\mathrm{UV}, \mathrm{He}$ II $\lambda 1640$ is similarly variable (see, for example, Krolik et al. 1991). Peterson et al. (1990) show that most of the flux variability of both the high-ionization UV lines and the Balmer lines in NGC 5548 is due to the broad, slightly blueshifted component. ${ }^{6}$ In an optically thick cloud most of the variation in the line flux of a recombination line is caused by changes in the size of the ionized regions as the ionizing flux varies. An optically thin cloud produces only small changes in the line flux of a recombination line from a fully ionized ion (such as $\mathrm{H}$ I and He II) because the Strömgren depth of the ionized regions is larger than the cloud size. Such lines actually decline slightly in flux as $U$ increases because the recombination coefficients decline at higher temperatures. The theoretical lack of variability of He II in an optically thin cloud is clearly shown in Figure $8 b$ in Shields et al. (1995). ${ }^{7}$ Optically thick models, on the other hand, produce strong He II flux variability, as is seen. Clouds that are optically thin in the Lyman continuum but with a $\mathrm{He}^{++}$Strömgren depth less than the cloud size will still produce strong $\mathrm{He}$ II variability, but the $\mathrm{He}^{++}$ comes at the expense of the $\mathrm{He}^{+}$so $\mathrm{He}$ i $\lambda 5876$ would decline as the continuum brightens. This has never been reported. Instead, like He II $\lambda 4686, \mathrm{He}_{\mathrm{I}} \lambda 5876$ is a highly variable VBLR line (see Fig. 2 in Collier et al. 1998). Because of the strong variability of the $\mathrm{He}$ I and He II lines we believe that the VBLR is not predominantly optically thin.

\section{THE C III]/C IV RATIO}

Brotherton et al. (1994) noted that observed values of C III]/C IV in the VBLR were higher than expected from their photoionization models. Our optically thin carbon-line model grid (Fig. 4a) shows that increasing $U$ actually leads to a decrease in $\mathrm{C}$ III]/C IV, compounding the discrepancy between observed and modeled ratios for the VBLR. However, our optically thick grid shows an insensitivity to changes in the line ratio as $U$ increases. The lowest optical depth model that can replicate the observed $W_{\lambda}$ of the broad component of Ly $\alpha$ (Fig. 4c) approaches being optically thick with respect to the carbon-line-forming gas. As mentioned in $\S 4$, this $\tau_{912}=1$ model allows for a combination of optically thin and thick clouds to generate the observed $\mathrm{Ly} \alpha / \mathrm{H} \alpha$ ratio, although only models of optically thick gas can reproduce the observed carbon-line ratios over a wide range of $U$. Without high values of $U$ it is difficult to reconcile the observed broad width of the $\mathrm{C}$ III] line with photoionization models' predictions, at

\footnotetext{
6 This variability should not be confused with object-to-object profile differences that tend to be in the lower velocity gas (see Francis et al. 1992 and Brotherton et al. 1994).

7 The slight rise of He II at low $U$ in their Fig. $8 b$ is because their "thin" model is thicker than ours and their $\mathrm{He}^{++}$zone does not extend to the back of the cloud for low $U$.
}

least for high-luminosity AGNs. Furthermore, the $\mathrm{C}$ III]/C IV ratios Brotherton et al. (1994) report for their ALS VBLR sample match the values for our optically thick model grid, yielding reasonable values of $U=0.05$ and $n_{\mathrm{H}}=5.0 \times 10^{9}$. Even if the $\mathrm{C}$ III]/C IV ratio in Brotherton et al. is too high, as a result of incomplete deblending, the optically thick models still allow for higher values of $U$ for any reasonable value of $\mathrm{C} \mathrm{III]/C} \mathrm{IV.} \mathrm{This}$ argues that the VBLR is better modeled with optically thick clouds, although we cannot discount a contribution from optically thin gas farther from the central engine. We do not expect this component to contribute much to the high-velocity wings.

\section{THE $\lambda 1400$ BLEND}

Optically thin and optically thick models predict very different $(\mathrm{Si}$ IV $+\mathrm{O}$ IV] $) / \mathrm{C}$ IV ratios. In the optically thin case, over the canonical ranges of $U$ and $n_{\mathrm{H}}$, the emission from both $\mathrm{Si}$ IV and $\mathrm{O}$ IV] is negligible compared to that of $\mathrm{C}$ IV, yielding only ( $\mathrm{Si} \mathrm{IV}+$ $\mathrm{O} \mathrm{IV}] \mathrm{)} / \mathrm{C} \mathrm{IV}=0.02$. One would not expect to see any contribution from the VBLR to the $\lambda 1400$ blend in observed spectra if the VBLR component is optically thin. Brotherton et al. (1994), however, give a value of 0.42 for the ( $\mathrm{Si}$ IV $+\mathrm{O}$ IV] $) / \mathrm{C}$ IV ratio for the ALS VBLR. Although our solar abundance optically thick model does not quite yield this ratio, its value of 0.28 is much closer than that of the optically thin model. Although we do not address the effect of enhanced abundances on all line ratios in his paper, we point out that the ratio measured by Brotherton et al. can be fit with a $5 Z_{\odot}$ optically thick model.

\section{DISCUSSION}

\subsection{Variability of Emission from High-Velocity Gas}

Following Ferland et al. (1990), a purported lack of variability of the VBLR flux in response to continuum variation has often been cited as an argument for an optically thin VBLR. However, the Mrk 590 variability Ferland et al. discuss is between only two epochs separated by 3 yr. Ferland et al. mention additional examples by Gondhalekar (1990), O’Brien et al. (1989), and Perez et al. (1989). The first two papers consider low S/N IUE spectra. Gondhalekar (1990) considers the C IV and Ly $\alpha$ variability of a number of quasars. While the difference spectra in some cases do seem to show a narrower peak to $\mathrm{C}$ IV, given the poor $\mathrm{S} / \mathrm{N}$ (which is even worse in the difference spectra) and uncertainties in continuum fitting, it is not clear how significant these are. There are also about an equal number of cases of the difference spectra being broader than the individual spectra. O'Brien et al. (1989) analyze IUE spectra (and some $\mathrm{H} \beta$ ) spectra of $3 \mathrm{C} 273$. Given the low $\mathrm{S} / \mathrm{N}$ it is not clear whether the wings of the line really do vary less in flux. Perez et al. (1989) do show some cases of the core of the emission line appearing to vary more than the wings, but the line in question is $\mathrm{Mg}$ II, and this cannot be taken as support for an optically thin VBLR, since Mg II arises from optically thick clouds. The more extensive reverberation-mapping campaigns of the last decade and a half give better insights into line profile variability. Two relevant results for the issue of VBLR variability are as follows:

1. The lag for high-ionization lines is shorter than for lower ionization lines because the gas closer to the center of the AGN is more highly ionized (Gaskell \& Sparke 1986; Krolik et al. 1991; Korista et al. 1995; Kriss et al. 2000, etc.).

2. There are long-term changes in the profiles of lines. These changes are unrelated to changes in the ionizing flux (Wanders \& Peterson 1996).

These results mean that (1) in comparing profiles allowance must be made for the differing time delays and (2) in looking for 
profile changes due to changing ionizing continuum levels caution is needed when comparing spectra separated by much more than the light crossing time. We believe that failure to allow for these two effects vitiates the result of the profile comparisons quoted in Ferland et al. (1990). For NGC 5548, for example, the He II lag is an order of magnitude smaller than the $\mathrm{H} \beta$ lag (Korista et al. 1995). Examples of the significant improvements in emission-line results obtained when lags are allowed for can be found in Pogge \& Peterson (1992) and Shields et al. (1995). Gaskell (1988) analyzed the extent of the high-velocity C IV wings in NGC 4151 in the well-sampled, short-term IUE monitoring campaign of Ulrich et al. (1984) and found that the extent of the wings correlated well with the continuum flux. It is clear that in NGC 4151 the VBLR is the most variable C IV component (see Fig. 1 in Ulrich et al. 1984 or Fig. 1 in Stoner et al. 1984). This finding agrees with the He II and He I VBLR variability discussed above.

The rms spectra offer a powerful test of whether the wings vary less than the cores of lines. Many AGNs have been subject to high-quality monitoring, especially in the optical, and many rms spectra are available. The key issue is whether the line wings are broader or narrower in the rms spectra compared with the mean spectra. For the 17 AGNs considered by Kaspi et al. (2000) the FWHMs of the mean and the rms spectra are very well correlated. We find an even tighter correlation for the FWHMs of the 19 objects given by Wandel et al. (1999). For the latter sample we find the $\mathrm{FWHM}_{\mathrm{rms}} / \mathrm{FWHM}_{\text {mean }}=1.07 \pm 0.4$ with $\mathrm{FWHM}_{\mathrm{rms}}$ being on average $230 \mathrm{~km} \mathrm{~s}^{-1}$ wider. For the Kaspi et al. (2000) sample we find $\mathrm{FWHM}_{\mathrm{rms}} / \mathrm{FWHM}_{\text {mean }}=0.89 \pm 0.7$. C. M. Gaskell et al. (2007, in preparation) made independent measures of $\mathrm{FWHM}_{\text {rms }}$ and $\mathrm{FWHM}_{\text {mean }}$ for half the Wandel et al. sample, and their $\mathrm{FWHM}_{\mathrm{rms}} / \mathrm{FWHM}_{\text {mean }}$ ratio is consistent with what we get from the Wandel et al. measurements. For the two objects in common between Wandel et al. (1999) and Kaspi et al. (2000) the $\mathrm{FWHM}_{\text {mean }}$ measurements agree well, but Kaspi et al. have $\mathrm{FWHM}_{\mathrm{rms}}$ too small by $1000 \mathrm{~km} \mathrm{~s}^{-1}$. This suggests systematic differences in how $\mathrm{FWHM}_{\text {rms }}$ is measured. Although $\mathrm{FWHM}_{\text {mean }}$ and $\mathrm{FWHM}_{\mathrm{rms}}$ are the same on average, for individual objects the differences can be greater than the measuring errors. We propose that these differences result from a combination of changes in the line flux due to ionizing continuum changes and the longer term changes in line profiles due to other factors. It is worth noting that both the mean and rms profiles are different for differing observing seasons (see Wanders \& Peterson 1996). The FWHM does not, of course, necessarily reflect differences in the wings. Inspection of the nine mean and rms $\mathrm{H} \beta$ spectra shown in Wanders (1997) shows that the high-velocity wings in most objects are as variable as the cores, although $\mathrm{He}$ II introduces uncertainty on the blue side and [O III] on the red. Mrk 590 in particular has high-velocity wings present in the rms spectrum. We conclude that rms spectra strongly support high variability in flux of the VBLR.

\subsection{Negative Responsivity?}

Sparke (1993) has proposed that the innermost gas in NGC 5548 is optically thin and has a negative responsivity. This potentially explains three curious results of the 1989 monitoring campaign, viz, that some of the line autocorrelation functions are broader than the continuum autocorrelation function, that some of the line-continuum cross-correlation functions rise faster than the continuum autocorrelation function, and that the $\mathrm{H} \beta$ transfer function does not peak at zero lag. Although this is an interesting idea, we do not believe it is tenable, because detailed photoionization models are unable to reproduce the required negative responsivity for the lines while producing enough line flux. When a cloud becomes optically thin (e.g., when the ionizing flux increases so that the Strömgren depth exceeds the size of the cloud) the Ly $\alpha$ flux ceases to rise. The He II line flux will continue to rise for another order of magnitude in $U$ as the size of the $\mathrm{He}^{++}$zone increases. When the $\mathrm{He}^{++}$zone equals the cloud size the He II flux will also cease to increase. $\mathrm{C}$ IV line flux rises with the He II flux, but while the He II flux plateaus, the $\mathrm{C}$ IV flux will fall rapidly after a further order of magnitude increase in $U$ because $\mathrm{C}^{+++}$is going to higher stages of ionization. All of these effects can be seen in Figure 1 of Shields et al. (1995). While it would appear that $\mathrm{C}$ IV can indeed show the negative responsivity needed for the Sparke (1993) model, this only occurs at a value of $U$ that is 2 orders of magnitude higher than the canonical value in the BLR. Under such conditions the cloud is very optically thin, and as we have noted in $\S 3$, the equivalent width of the lines produced by the gas is too small. Thus, any gas with a negative responsivity is going to produce a negligible contribution to the total line flux.

We suggest that the differences in the slopes and widths of the correlation functions are on the order of the errors in these functions. The noisier the measurements, the more peaked the autocorrelation function is, and the line fluxes have higher uncertainties than the continuum intensity. In the limit of infinite noise one gets the sampling-window autocorrelation function (see Gaskell \& Peterson 1987). This effect is obvious in the Mg II autocorrelation function in Figure 1 in Sparke (1993), where there is a clear spike at the origin, although, in general, the uncertainties in the correlation functions will mask the narrow spike. Inspection of additional correlation functions support this interpretation. In Figure 14 in Korista et al. (1995), for example, the width of the optical-UV cross-correlation function is narrower both at half maximum and at zero than the autocorrelation function of the UV alone. A negative responsivity of the optical emission to changes in the $\mathrm{UV}$ is not a physically viable explanation of this effect. If the line autocorrelation functions really are broader than the continuum autocorrelation function (which we do not believe), one possible explanation could be that the observed UV/optical continuum is itself reprocessed from the photoionizing continuum. Multiwavelength observations of NGC 7469 (Wanders et al. 1997; Collier et al. 1998) show that there is such continuum reprocessing going on in at least NGC 7469. The failure of the NGC $5548 \mathrm{H} \beta$ transfer function to peak at zero (see Horne et al. 1991), on the other hand, is highly significant and must be real. We suggest that the explanation of this is the one offered by Horne et al. (1991): that the $\mathrm{H} \beta$-emitting gas is not spherically symmetric. Since the Balmerline profiles of NGC 5548 show prominent structure and we believe such structure is due to a disk component (Gaskell \& Snedden 1999), we consider this very likely.

\subsection{An Optically Thin Contribution to the General BLR?}

While we have argued that the VBLR is not predominantly optically thin, it would be highly unlikely if there were not at least some optically thin clouds in both the VBLR and the rest of the BLR. A real BLR doubtless consists of clouds with a wide range of sizes - perhaps with a fractal structure, as suggested by Bottorff \& Ferland (2001). The change in line ratios as the continuum varies probably provides the best evidence for this possibility. Wamsteker \& Colina (1986) found that the normal rapid rise of C IV flux with continuum level in Fairall 9 appeared to stop when the continuum reached a high level. The effect was less pronounced for Ly $\alpha$. They suggested that the effect was due to clouds making a transition from being radiation bounded to being matter bounded (i.e., optically thin). This effect has been thoroughly studied by Shields et al. (1995). Allowing for the lags in the C IV and Ly $\alpha$ emission removes the abruptness of the effect, but the $\mathrm{C}$ Iv/ Ly $\alpha$ 
ratio still declines at high luminosities. Like Shields et al. (1995), we are unable to reproduce this behavior with a purely optically thick model, and we concur that an optically thin contribution is needed as well.

\section{CONCLUSIONS}

We conclude that rather than being optically thin, the highvelocity BLR gas is predominantly optically thick. We believe this because of the following properties of the high-velocity gas:

1. The equivalent widths calculated from optically thin photoionization models are too small.

2. The observed $\mathrm{Ly} \alpha / \mathrm{H} \alpha$ ratios are best matched by optically thick photoionization models.

3. The Balmer decrements derived from optically thick models better match the observed values in the high-velocity wings of $\mathrm{H} \alpha$ and $\mathrm{H} \beta$.

4. One would expect to see a difference between high- and low-ionization line profiles if there were a significant optically thin high-velocity component of the line-producing gas. There is no strong evidence of this.
5. The strong variability in flux of the high-ionization lines cannot be explained with optically thin clouds.

6. The observed $\mathrm{C}$ III]/C IV ratios are best fit with optically thick models.

7. Optically thick models predict a value for $(\mathrm{Si}$ IV $+\mathrm{O}$ IV $]) / \mathrm{C}$ IV that is more consistent with observed values.

If the high-velocity gas is predominantly optically thick, it is thus not fundamentally different, in terms of optical depth, from the lower velocity gas.

We are grateful to Giovanna Stirpe for making her optical spectra available in machine-readable format. We also wish to acknowledge very helpful and detailed comments by Mark Bottorff, Liz Klimek, Kirk Korista, Joe Shields, and Bev Wills. This work has been supported in part by grant AR-05796.01-94A from the Space Telescope Science Institute, which is operated by the Association of Universities for Research in Astronomy (AURA), Inc., under NASA contract NAS5-26555 and by the National Science Foundation (NSF) through grant AST 03-07912.
Baldwin, J., Ferland, G., Korista, K., \& Verner, D. 1995, ApJ, 455, L119

Bechtold, J., Shields, J., Rieke, M., Ji, P., Scott, J., Kuhn, O., Elvis, M., \& Elston, R. 1997, in ASP Conf. Ser. 113, Emission Lines in Active Galaxies: New Methods and Techniques, ed. B. M. Peterson, F. Cheng, \& A. S. Wilson (San Francisco: ASP), 122

Boroson, T. A., \& Green, R. F. 1992, ApJS, 80, 109

Bottorff, M. C., \& Ferland, G. J. 2001, ApJ, 549, 118

Bottorff, M. C., Korista, K. T., Shlosman, I., \& Blandford, R. D. 1997, ApJ, 479,200

Brotherton, M. S., Wills, B. J., Francis, P. J., \& Steidel, C. C. 1994, ApJ, 430, 495

Burstein, D., \& Heiles, C. E. 1982, AJ, 87, 1165

Chiang, J., \& Murray, N. 1996, ApJ, 466, 704

Collier, S. J., et al. 1998, ApJ, 500, 162

Collin-Souffrin, S., \& Lasota, J.-P. 1988, PASP, 100, 1041

Crenshaw, D. M. 1986, ApJS, 62, 821

Davidson, K. 1977, ApJ, 218, 20

De Zotti, G., \& Gaskell, C. M. 1985, A\&A, 147, 1

Espey, B. R., et al. 1994, ApJ, 434, 484

Ferland, G. J. 2002, HAZY, A Brief Introduction to CLOUDY C90.04 (Dept.

Phys. Astron. Internal Rep.; Lexington: Univ. Kentucky)

Ferland, G. J., Korista, K. T., \& Peterson, B. M. 1990, ApJ, 363, L21

Ferland, G. J., \& Persson, S. E. 1989, ApJ, 347, 656

Francis, P. J., Hewett, P. C., Foltz, C. B., \& Chafee, F. H. 1992, ApJ, 398, 476

Gaskell, C. M. 1982, ApJ, 263, 79

. 1987, in Astrophysical Jets and Their Engines, ed. W. Kundt (Dordrecht: Reidel), 21

$$
\text { . 1988, ApJ, 325, } 114
$$

2000, NewA Rev., 44, 563

Gaskell, C. M., \& Benker, A. J. 2007, ApJ, submitted

Gaskell, C. M., Goosmann, R. W., Antonucci, R. R. J., \& Whysong, D. 2004, ApJ, 616, 147

Gaskell, C. M., \& Mariupolskaya, V. Y. 2002, in ASP Conf. Ser. 255, Mass Outflow in Active Galactic Nuclei: New Perspectives, ed. D. M. Crenshaw, S. B. Kraemer, \& I. M. George (San Francisco: ASP), 261

Gaskell, C. M., \& Peterson, B. M. 1987, ApJS, 65, 1

Gaskell, C. M., \& Snedden, S. A. 1999, in ASP Conf. Ser. 175, Structure and Kinematics of Quasar Broad Line Regions, ed. C. M. Gaskell, W. N. Brandt, M. Dietrich, D. Dultzin-Hacyan, \& M. Eracleous (San Francisco: ASP), 157

Gaskell, C. M., \& Sparke, L. S. 1986, ApJ, 305, 175

Gondhalekar, P. M. 1990, MNRAS, 243, 443

Grandi, S. A., \& Phillips, M. M. 1979, ApJ, 232, 659

Horne, K., Welsh, W. F., \& Peterson, B. M. 1991, ApJ, 367, L5

Kaspi, S., Smith, P. S., Netzer, H., Maoz, D., Jannuzi, B. T., \& Giveon, U. 2000, ApJ, 533, 631

Koratkar, A. P., \& Gaskell, C. M. 1991, ApJS, 75, 719

Korista, K. T., \& Goad, M. R. 2004, ApJ, 606, 749

Korista, K. T., et al. 1995, ApJS, 97, 285

Kriss, G. A., Peterson, B. M. Crenshaw, D. M., \& Zheng, W. 2000, ApJ, 535, 58

Krolik, J. H., Horne, K., Kallman, T. R., Malkan, M. A., Edelson, R. A., \& Kriss, G. A. 1991, ApJ, 371, 541

\section{EFERENCES}

Marziani, P., Sulentic, J. W., Zamanov, R., \& Calvani, M. 2003, Mem. Soc. Astron. Italiana, 74, 490

Mathews, W. G., \& Wampler, E. J. 1985, PASP, 97, 966

Morris, S. L., \& Ward, M. J. 1989, ApJ, 340, 713

Mushotzky, R. F., \& Ferland, G. J. 1984, ApJ, 278, 558

Netzer, H. Brotherton, M. S., Wills, B. J., Han, M.-S., Wills, D., Baldwin, J. A.,

Ferland, G. J., \& Browne, I. W. A. 1995, ApJ, 448, 27

O’Brien, P. T., Wilson, R., \& Zheng, W. 1989, MNRAS, 240, 741

Osterbrock, D. E. 1977, ApJ, 215, 733

- 1989, Astrophysics of Gaseous Nebulae and Active Galactic Nuclei (Mill Valley: University Science Books)

Osterbrock, D. E., \& Shuder, J. M. 1982, ApJS, 49, 149

Perez, E., Penston, M. V., \& Moles, M. 1989, MNRAS, 239, 75P

Peterson, B. M., Reichert, G. A., Korista, K. T., \& Wagner, R. M. 1990, ApJ, 352,68

Peterson, B. M., Wanders, I., Bertram, R., Hunley, J. F., Pogge, R. W., \& Wagner, R. M. 1998, ApJ, 501, 82

Pogge, R. W., \& Peterson, B. M. 1992, AJ, 103, 1084

Rodriguez-Ardila, A., Pastoriza, M. G., \& Donzelli, C. J. 2000, ApJS, 126, 63

Schlegel, D. J., Finkbeiner, D. P., \& Davis, M. 1998, ApJ, 500, 525

Shields, J. C., Ferland, G. J., \& Peterson, B. M. 1995, ApJ, 441, 507

Shuder, J. M. 1982, ApJ, 259, 48

$$
\text { 1984, ApJ, 280, } 491
$$

Snedden, S. A., \& Gaskell, C. M. 1997, in ASP Conf. Ser. 113, Emission Lines in Active Galaxies: New Methods and Techniques, ed. B. M. Peterson, F.-Z.

Cheng, \& A. S. Wilson (San Francisco: ASP), 195

- 1999a, ApJ, 521, L91

1999b, in ASP Conf. Ser. 175, Structure and Kinematics of Quasar Broad

Line Regions, ed. C. M. Gaskell, W. N. Brandt, M. Dietrich, D. Dultzin-Hacyan,

\& M. Eracleous (San Francisco: ASP), 25

Sparke, L. S. 1993, ApJ, 404, 570

Stirpe, G. M. 1990, A\&AS, 85, 1049 . 1991, A\&A, 247, 3

Stoner, R., Ptak, R., \& Gregory, S. 1984, ApJ, 285, 69

Sulentic, J. W., Marziani, P., \& Dultzin-Hacyan, D. 2000, ARA\&A, 38, 521

Ulrich, M.-H., et al. 1984, MNRAS, 206, 221

Vanden Berk, D. E., et al. 2001, AJ, 122, 549

Wamsteker, W., \& Colina, L. 1986, ApJ, 311, 617

Wandel, A., Peterson, B. M., \& Malkan, M. A. 1999, ApJ, 526, 579

Wanders, I. 1997, in IAU Colloq. 159, Emission Lines in Active Galaxies: New Methods and Techniques, ed. B. M. Peterson, F.-Z. Cheng, \& A. S. Wilson (ASP Conf. Ser. 113; San Francisco: ASP), 183

Wanders, I., \& Peterson, B. M. 1996, ApJ, 466, 174

Wanders, I., et al. 1997, ApJS, 113, 69

Ward, M. J., \& Morris, S. L. 1984, MNRAS, 207, 867

Wilkes, B. J. 1984, MNRAS, 207, 73

Wilkes, B. J., \& Carswell, R. F. 1982, MNRAS, 201, 645

Wills, B. J., Netzer, H., Brotherton, M. S., Han, M.-S., Wills, D., Baldwin, J. A.,

Ferland, G. J., \& Browne, I. W. A. 1993, ApJ, 410, 534

Zheng, W. 1992, ApJ, 385, 127 